\title{
Entrevista a Jorge Bertheau
}

\author{
Jorge Grané - 22 octubre 2016
}

Invitado nacional

Universidad de Costa Rica

jorgegrane@gmail.com

Recibido: 27 de abril 2016

Aceptado: 31 de marzo 2017

\section{Jorge Bertheau}

Obtuvo su título de Arquitecto en la Universidad Nacional Autónoma de México (UNAM), en 1965. Fue diplomado en Métodos Educativos en Enseñanza Universitaria de The Architectura Association, School of Architecture de Londres. Su labor docente la realizó desde 1964 a 1991 en la Facultad de Bellas Artes y la Escuela de Arquitectura de la Universidad de Costa Rica (UCR). Fue cofundador de dicha Escuela en los años 70s. En esta Escuela fue nombrado subdirector y coordinador académico entre 1971 y 1981 y director en los años de 1989 a 1991. Jorge Bertheau es uno de los tres profesores eméritos de la Escuela de Arquitectura.

\section{Jorge Grané}

Licenciatura en Periodismo, UACA, Costa Rica 1991. Licenciatura en Arquitectura, Argentina, 1967 Docente, Escuela de Arquitectura de la Universidad de Costa Rica (UCR).

\section{Resumen:}

Jorge Bertheau construyó su casa de ladrillo, en Escazú, hace 35 años. Estudió Arquitectura en México y, a su regreso, trabajó en el estudio de Rafael "Felo" García con quien entabló una profunda amistad. Más adelante, Edgar Brenes se unió a ellos y juntos gestaron la idea de fundar la Escuela de Arquitectura de la Universidad de Costa Rica (UCR), la que tuvo poco apoyo por parte de las autoridades universitarias.

Con una beca ofrecida por Inglaterra, Jorge Bertheau y Felo García fueron a ese país a conocer programas de estudio. Más tarde se les unió Edgar Brenes.

A su regreso, en 1971, se abrió la Escuela, con la consigna de "aprender a aprender". La flamante Escuela tuvo detractores y seguidores. Entre estos últimos se destacó la figura del Ingeniero Walter Sagot.

A la lucha por la supervivencia de la Escuela, Bertheau la califica como "el momento heroico" de la misma, que luego se diluyó con otra gente y otras ideas.

Muchos profesores se acercaron, en sus inicios, con entusiasmo, para apoyar la misión de la Escuela y así se formaron, con éxito los primeros arquitectos costarricenses.

Palabras clave: aprender a aprender; fundación de la Escuela; momento heroico; primeros arquitectos.

\section{Jorge Bertheau interview}

\section{Abstract:}

Jorge Bertheau built his brick house in Escazú, 35 years ago. He studied Architecture in Mexico and, upon his return, worked in Rafael "Felo" Garcia's design studio with whom he established a deep friendship. Later, Edgar Brenes joined them and together they initiated of founding the School of Architecture, which had little support from the university authorities.

With a scholarship offered by England, Jorge Bertheau and Felo García went there to learn and understand study programs. Later on Edgar Brenes joined them.

Upon his return, in 1971, the School was opened, with the "learning to learn" slogan. The new School had detractors and followers. Among the latter was the figure of Engineer Walter Sagot.

Bertheau describes the struggle for the survival of the School as "the heroic moment", which later diluted with other people and other ideas.

Many teachers approached, in the beginning, with enthusiasm, to support the mission of the School and thus the first Costa Rican architects graduated with success.

Keywords: learning to learn; foundation of the School; heroic momento; first architects. 


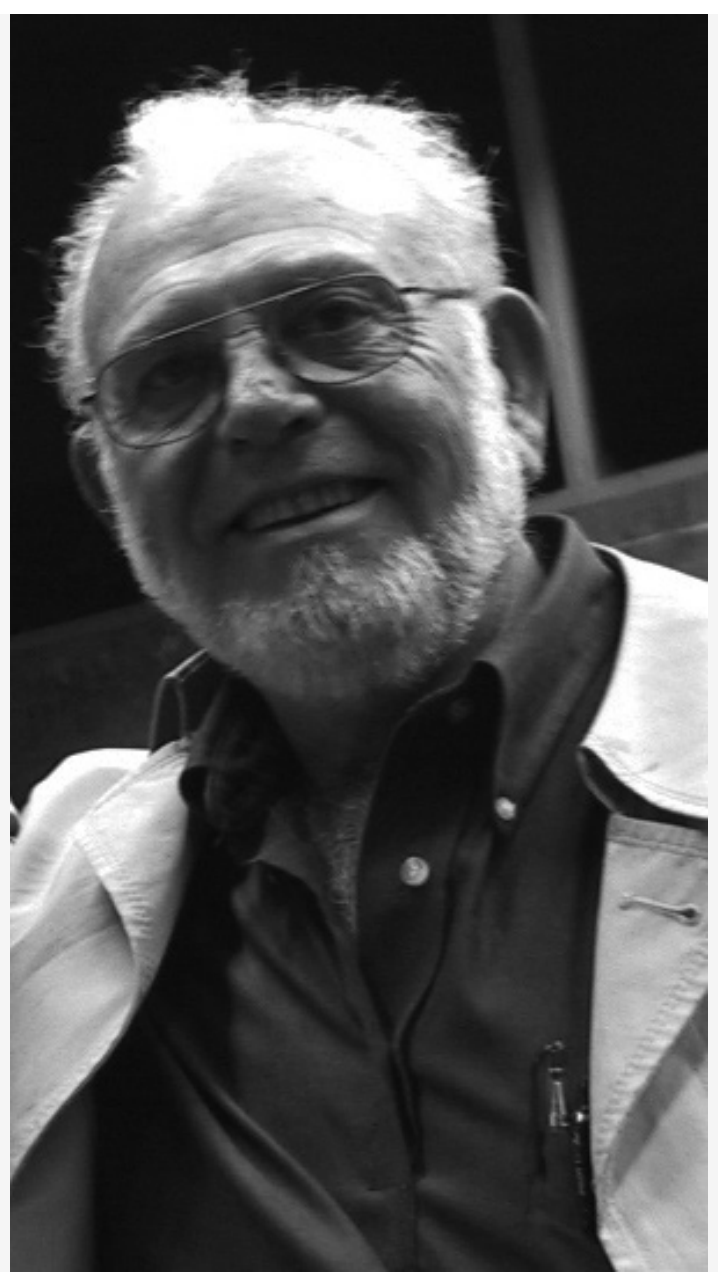

\section{$v$}

orge Bertheau vive en un lugar de Escazú que no quiere urbanizarse. Su jardín conserva la vocación de un bosque antiguo, y allí se oye un murmullo de agua que es el lamento del río Chiquero.

Ahí construyó su casa, sin planos municipales, con ladrillos que le sobraron a un amigo y la complicidad de un albañil perfeccionista que no duró en el trabajo.

Fue hace 35 años y ese era el lugar de la casa de la artista Margarita Bertheau, su madre, de la cual rescató, en la demolición, material de construcción que luego usó en estructuras de pochote o en bibliotecas donde caben títulos de Henri Marcuse, Karl Marx o Fabián Dobles.

Con confesas influencias de Wright y un librito sobre arcos, de un arquitecto español, trazó en obra, con un clavo y una soga, círculos gigantes de ladrillos, y otros más pequeños, que dividen los espacios de la casa. Ya en Costa Rica desaparecía el uso del ladrillo y se imponía el bloque de concreto en la construcción.

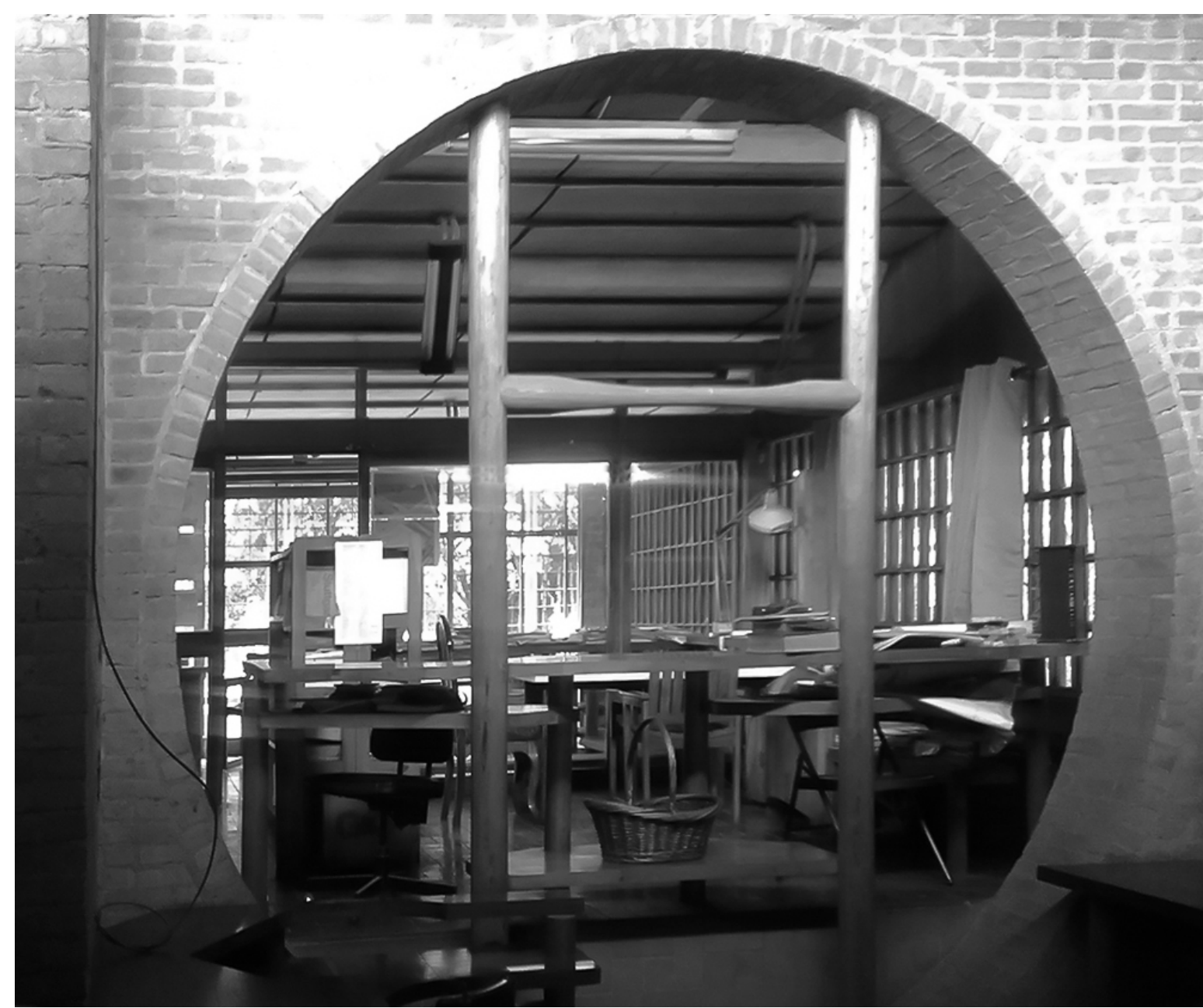


En el año 1957, Jorge se trasladó a México para cursar la carrera de Arquitectura, destino preferido por quienes, con vocación de arquitectos, no tenían la posibilidad de estudiar en su propio país.

A su regreso a Costa Rica fue invitado por el arquitecto Rafael "Felo" García para integrarse a su estudio. Jorge Bertheau recuerda ese encuentro como un momento mágico de sincronía entre dos personas que luego cambiarían la historia de la Arquitectura en Costa Rica.

En poco tiempo coincidieron en que debían hacer, juntos, algo para que en el país se fundara la Escuela de Arquitectura y otras cosas más: separarse de los ingenieros, quitar los timbres de construcción o remodelar la ciudad de San José.

Acepta, Jorge, que no pudieron lograr todo, pero crearon un fuerte espíritu de grupo con otros arquitectos que fueron sumándose a sus emprendimientos. Fueron ellos los que propiciaron la peatonalización de la Avenida Central. También propusieron la demolición de la Catedral y el Parque Central para hacer un estacionamiento subterráneo y un nuevo templo metropolitano, pero no fueron comprendidos por la curia.

Fueron jurados, Jorge Bertheau y Felo García, en el concurso del edificio del Colegio de Ingenieros Agrónomos, que ganó Edgar Brenes sobre Franz Beer. Así se conocieron con Edgar quien, con entusiasmo, se integró al grupo de los emprendedores.

Propusieron una Avenida Central en Guadalupe, Moravia y Tibás, y construir un parqueo de tres niveles bajo la Avenida Segunda.

Repartían, en varios frentes, copias con sus ideas, hechas en un polígrafo de alcohol, apoyando o rechazando propuestas, como la creación del Colegio de Ingenieros y de Arquitectos, donde los Arquitectos siempre serán minoría.

Llegaron, así, a organizar la Primera Semana de la Arquitectura, que se llevó a cabo en la Facultad de Educación, con invitados de países de América y Europa. Así llegaron el urbanista L. Noviant, de Francia, el arquitecto W. Chambers, de Inglaterra y Ricardo Legorreta, de México. La intención era llamar la atención sobre la propuesta de fundar la Escuela de Arquitectura y pedir apoyo externo para su concreción.

El intento tuvo eco ya que la Universidad decidió formar una Comisión para estudiar la posibilidad de crear dicha Escuela en la sede Rodrigo Facio. Jorge Bertheau no fue invitado a participar en las deliberaciones pero luego, a pedido de Felo García y Edgar Vargas, se integró a la Comisión.

Se creó una buena relación con Inglaterra y así fue cómo el Director de postgrado de la Architectural Association (AA), el arquitecto Otto Köningsberger visitó el país para interiorizarse de las ideas del grupo promotor de la Escuela. Entonces, desde Londres, surgió la iniciativa de ofrecer 12 becas para que arquitectos ticos se informaran, en Inglaterra, de cómo se llevaba a cabo la educación de los estudiantes ingleses.

Los primeros en viajar fueron Jorge y Felo, con la beca, junto con Edgar Brenes quien los acompañó. Desde ese momento, bromea Jorge, para la $U$, el centro del mal estaba en Londres.

Anteriormente habían visitado las Escuelas de Arquitectura de Centroamérica así como la de Zulia, en Venezuela y Córdoba, en Argentina. Hay que recordar que eran los tiempos revueltos posteriores a la revolución cultural de mayo del 68, en Paris, cuyo slogan era "la imaginación al poder". El terreno estaba abonado para que las ideas de cambio fructificaran también en Latinoamérica.

Los tres, Felo, Edgar y Jorge acudieron a la Architectural Association donde se matricularon para obtener el título en Teaching Methods for Higher Education. Los dos primeros dominaban el inglés mientras que Jorge se la jugaba con ese idioma.

Parte del curso consistía en compartir ideas y charlas con diversas personalidades que visitaban Londres. Escogían, de una lista de arquitectos, sociólogos o urbanistas, a los que preferían conocer para discutir los temas de su interés, y así pasaban, con ellos, dos o tres días de aprendizaje, entre conversaciones y tragos. 
Para trabajar usaban una gran pizarra donde anotaban sus ideas, las que compartían con los profesores de la $A A$.

Durante la presentación de graduación, el grupo tico quebró las rígidas reglas inglesas y ofreció una copa de vino al público concurrente, anticipándose al brindis final.

A todo esto, la Comisión Universitaria hizo un amago de programa tradicional donde coexistían el cálculo, la electricidad, la construcción, junto a la sociología, la teoría, la historia, que se sumaba al trabajo de los estudiantes en Taller.

Según Jorge Bertheau, pretender abarcar todos estos conocimientos, en cinco años, era una payasada. Lo que debía enseñarse en Arquitectura era "aprender a aprender", o sea, inculcar las necesarias cualidades para saber dónde encontrar la información adecuada para solucionar los problemas que se presentan: primero en la carrera y después, en la profesión. La meta era crear una base sólida de conocimiento y, desde allí, aprender a investigar las situaciones dadas.

Estas propuestas surgían de los estudios sobre la educación moderna que negaban todo lo que fuera lo clásico-tradicional. Jorge Bertheau insiste en repetir la frase: entre más impredecibles los resultados, mejor es la educación.

Una vez fundada la Escuela de Arquitectura, en 1971, la Universidad solicitaba, todos los años, que se diera a conocer el "perfil" del Arquitecto, lo cual nunca cumplieron.

En cuanto a la fase pedagógica, se sustituyeron las materias antiguas por otras novedosas como Teoría de Modificación del Entorno o Arquisistemas, donde se trataban temas como sistemas complejos altamente probabilísticos de aproximación a la naturaleza y a los fenómenos naturales.

El Consejo Universitario, preocupado por estas innovaciones inconsultas, pidió la opinión del Rector de la Universidad de Puerto Rico, Ismael Rodríguez Bou, quien empezó mirando el programa con desconfianza y acabó apoyándolo con entusiasmo.

También llamaron al arquitecto Javier Carvajal Ferrer para evaluar el proceso académico de la Escuela y nombraron al Ing. Walter Sagot como mediador en la controversia, quien no parecía convencido de los resultados de las nuevas propuestas.

Una noche, saliendo Bertheau, Brenes y García del bar Chelles, en la Avenida Central, se encontraron con Sagot y un grupo de ingenieros recién egresados con quienes empezaron a discutir sobre el tema que los distanciaba. Para resolver la disputa, decidieron ir al bar Ye pub, propiedad de Edgar Brenes, para enfrentarse a un duelo de cervezas, las que se servían en unos vasos de vidrio de una yarda de largo. Beber, sin emborracharse, requería de habilidades y estrategias que los arquitectos conocían bien, y fueron declarados vencedores.

Desde ese momento, y como buen perdedor, Walter Sagot se unió, como amigo, al grupo de Arquitectura y se convirtió, según palabras de Jorge Bertheau y opinión de Edgar Brenes, en el bastión defensor de la Escuela.

Cuenta Jorge que el Ing. Sagot les advirtió, una vez, que corría el rumor que en la Escuela de Arquitectura se sembraba marihuana. Entonces, Louis Ducoudray, Edgar Brenes, el propio Jorge Bertheau y otros voluntarios, se vistieron con pantalones cortos y echaron agua, con regaderitas, a unas macetas en la puerta de la Escuela, a la vista de los universitarios que por allí pasaban.

En otra ocasión, en que el Consejo Universitario se había reunido con un arquitecto español para evaluar, una vez más, la labor de los profesores, el Ing. Sagot sugirió ir a conocer la obra de los arquitectos cuestionados, los cuales salieron airosos de la prueba.

La lucha que libraron todo ese tiempo, Jorge Bertheau la califica como "el momento heroico" de la Escuela, que fue luego perdiendo fuerza con la necesaria llegada de otros protagonistas, menos comprometidos con lo que ya era una realidad.

El arquitecto Louis Ducoudray fue una importante figura en el proceso, por sus saberes sobre la Teoría de Sistemas, que a todos les fascinaba. Mario Lungo se destacaba por sus conocimientos de epistemología que había adquirido en La Sorbona. Juan Bernal Ponce, arquitecto chileno, montó un Taller de Grabado que todavía se recuerda. Se 
Edificio de la Escuela de Arquitectura, 2015. Foto de Rodolfo M. Granados

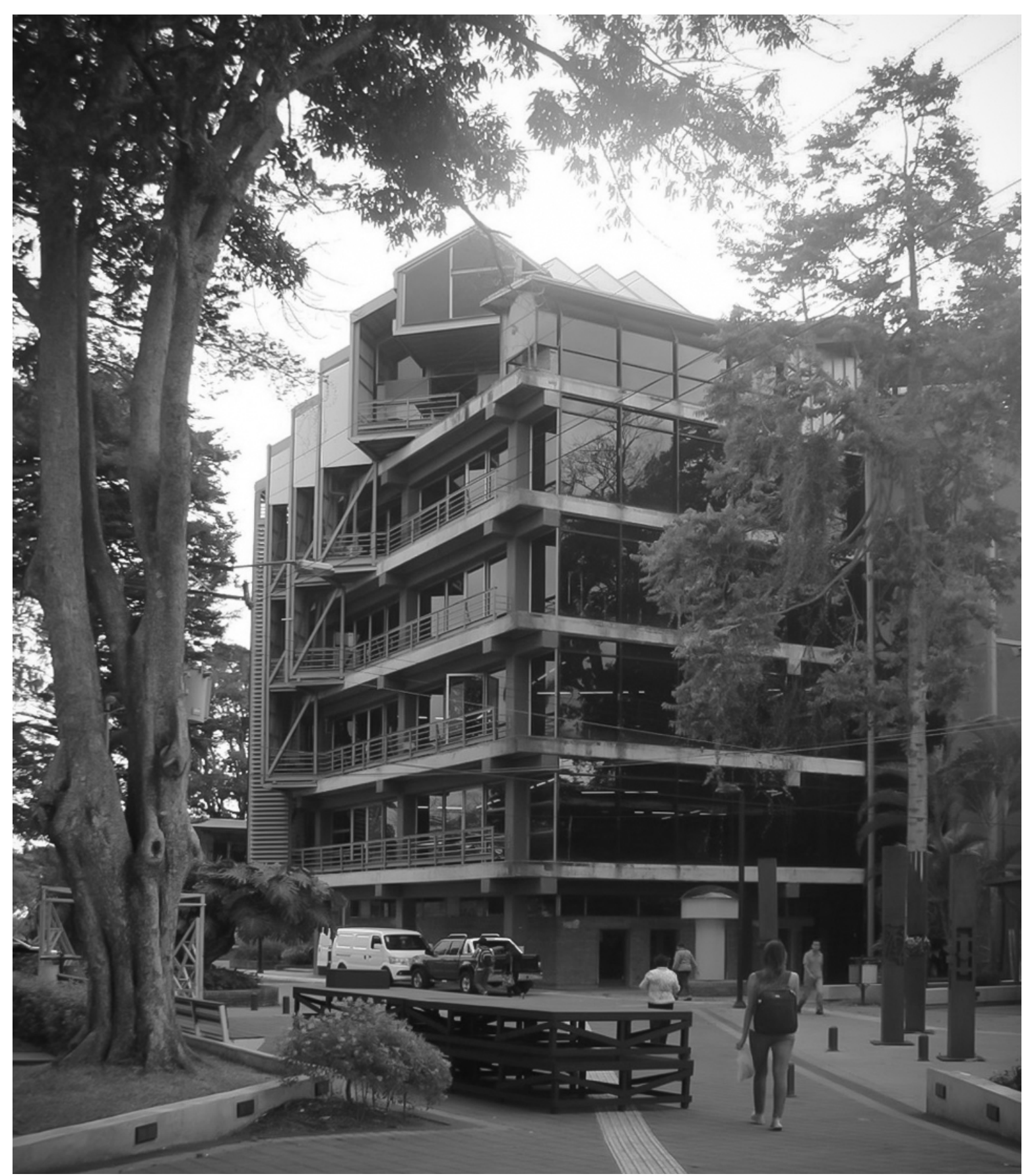

unieron a ellos, Meza, Aristizabal, Pedro Rey, Franz Beer y, después, la ingeniera Nora Brenes a quien sus colegas ingenieros le reclamaban su amistad con los Arquitectos.

Hay que destacar que los estudiantes respondían con gran voluntad al rigor de una experiencia nueva y, a veces, incomprendida. También a ellos, como Manuel Morales, Ofelia Sanou o Jorge "Pino" Castro, hay que darles el crédito de haber sufrido, confiado y disfrutado su paso por la Escuela.

Pronto el edificio se llenó de estudiantes y así llegaron otros profesores, con propuestas nuevas. Esto coincidió con el despertar de ideologías convulsas, con ideas para las que no estaba preparada la Escuela.

Dice Jorge que estas nuevas caras ya no fueron parte del "momento heroico", el cual, para él, ya había muerto.

Un largo par de horas estuvo Jorge hablando en la sala de su casa. Lo que parecía que iba a ser una entrevista fue un relato ininterrumpido, siempre con el mismo entusiasmo, en el que Jorge contó lo bueno y lo malo de la lucha por conseguir que la Escuela de Arquitectura, que hoy disfrutamos, se hiciera realidad.

Lo destacable de esa lucha no fue tanto el logro sino el ejemplo de estos Arquitectos por defender sus ideas y sus convicciones ante quienes se oponían al cambio.

Fue triste y lapidaria la frase de despedida de Jorge: Costa Rica le tiene miedo a cualquier pensamiento nuevo. 